\title{
Konjunkturschlaglicht
}

\section{COVID-19 und die Wirtschaft in Afrika}

Die Corona-Pandemie hat auch Afrika ergriffen. Allerdings sind die offiziell erfassten Zahlen der Infektionen und der Todesfälle vergleichsweise gering. In Afrika wurden mit 7,5 Mio. lediglich 3,5\% aller COVID-19-Fälle und mit knapp 200.000 4,3\% aller coronabedingten Todesfälle weltweit registriert, obwohl der Kontinent fast $18 \%$ der Weltbevölkerung ausmacht.

\section{Unterschätzte Evidenz}

Die relativ niedrigen Infektionszahlen in Afrika lassen sich nicht durch die politischen Reaktionen auf die Pandemie erklären. Zwar verhängten auch in Afrika viele Regierungen in der Anfangsphase der Pandemie umgehend Maßnahmen zu ihrer Eindämmung. Die Strenge variierte jedoch von Land zu Land, und die Eindämmungsmaßnahmen erwiesen sich oft als schwierig durchzusetzen. Eine Reihe afrikaspezifischer Faktoren, wie die junge Bevölkerung - nur 5,5\% der Menschen in Afrika sind älter als 60 Jahre, verglichen mit rund $25 \%$ in Europa und Nordamerika -, wurde zur Erklärung der niedrigen Infektions- und Sterblichkeitsraten auf dem Kontinent vorgeschlagen, aber sie reichen wohl nicht aus.

Wesentlich sind auch die begrenzten Test- und Überwachungskapazitäten in vielen Ländern, die zu einer gravierenden Untererfassung der Pandemie führen. Hierfür spricht, dass sich die Hälfte der Fälle auf dem Kontinent auf die drei relativ weit entwickelten Länder Südafrika, Marokko und Tunesien konzentriert, die nur $8 \%$ seiner Bevölkerung ausmachen. Gleichzeitig meldete Nigeria, das bevölkerungsreichste Land Afrikas, bislang nie mehr als 5 Infektionen pro 100.000 Einwohner:innen, während eine Studie auf der Grundlage von Blutproben aus Lagos ergab, dass $23 \%$ der Bevölkerung infiziert waren (Bloomberg, 2021). Daten zur Übersterblichkeit - die Zahl der tatsächlich eingetretenen Todesfälle im Vergleich zu der Zahl, die ohne COVID-19 zu erwarten gewesen wäre - sind für afrikanische Länder im Allgemeinen nicht aus

(C) Der/die Autor:in 2021. Open Access: Dieser Artikel wird unter der Creative Commons Namensnennung 4.0 International Lizenz veröffentlicht (creativecommons.org/licenses/by/4.0/deed.de).

Open Access wird durch die ZBW - Leibniz-Informationszentrum Wirtschaft gefördert.

1 Es werden unter anderem wärmere Temperaturen, eine relativ geringe Mobilität der Bevölkerung sowie genetische, immunologische und komorbide Faktoren diskutiert, vgl. z. B. Tessema und Nkengasong (2021). offiziellen Quellen verfügbar. Die Zeitschrift The Economist (2021) hat dazu Schätzungen für eine große Zahl von Ländern erstellt. Demnach sind in Afrika zwischen 62 und 160 Todesfälle pro 100.000 Menschen auf die Pandemie zurückzuführen, gegenüber einer Quote von 14,3 offiziell gemeldeten COVID-19-bedingten Todesfällen (vgl. Abbildung 1). Zwar ist die Übersterblichkeit damit in Afrika im Vergleich mit anderen Weltregionen immer noch relativ gering, doch ist die Dunkelziffer besonders hoch.

\section{Gravierende wirtschaftliche Auswirkungen}

Infolge der Pandemie brach auch in Afrika die Produktion 2020 ein. Der Rückgang des Bruttoinlandsprodukts (BIP) war zwar weniger stark als ursprünglich befürchtet und fiel geringer aus als in den fortgeschrittenen Volkswirtschaften oder in Lateinamerika. Gleichwohl wurde nach Angaben der Weltbank mit -1,8\% im Jahresdurchschnitt immer noch der historisch stärkste Einbruch des BIP und die erste Rezession seit 25 Jahren verzeichnet. Die Auswirkungen sind von Land zu Land unterschiedlich und waren in Süd- und Nordafrika am stärksten (vgl. Abbildung 2). Einfache Korrelationsanalysen deuten darauf hin, dass die wirtschaftlichen Folgen in Ländern mit einer hohen Abhängigkeit von Rohstoffen, Tourismus und internationalem Handel gravierender waren (Gern et al., im Erscheinen). Insgesamt sind Schätzungen zufolge rund 29 Mio. Menschen in Afrika wegen der Pandemie zusätzlich von extremer Armut betrof-

\section{Abbildung 1}

Übersterblichkeit nach Weltregionen

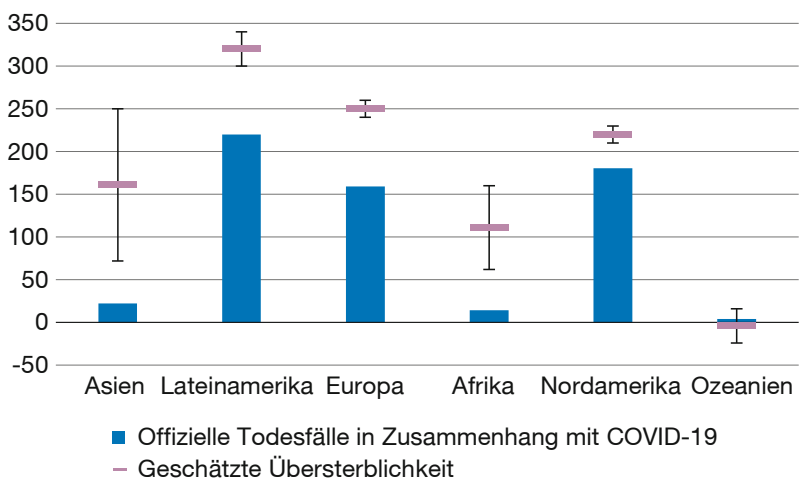

Anmerkungen: Jeweils pro 100.000 Menschen. Die rosa Balken geben die mittlere Schätzung je Region an; die schwarzen Bänder die Schätzunsicherheit.

Quelle: The Economist (2021). 


\section{Abbildung 2}

Bruttoinlandsprodukt in afrikanischen Ländern, 2020

Veränderung gegenüber 2019 in \%
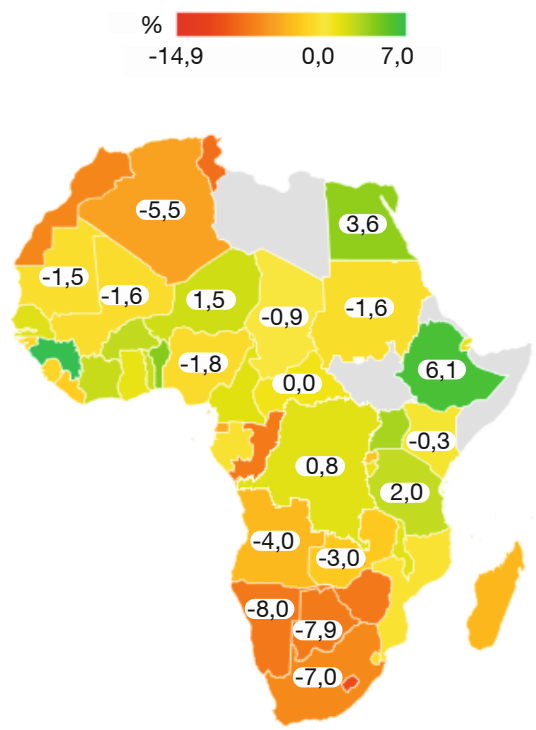

Unterstützt von Bing (c) GeoNames, Microsoft, TomTom

Quelle: Weltbank (2021)

fen (Mahler et al., 2021). Für 2021 wird für die afrikanischen Länder südlich der Sahara mit einem Produktionsanstieg von 3,4 \% gerechnet, was im internationalen Vergleich sehr wenig ist, während für Nordafrika ein Zuwachs um 7,3\% erwartet wird. Auf Pro-Kopf-Basis wird das BIP in Afrika voraussichtlich erst nach 2022 und in vielen Ländern nicht vor 2025 das Vorkrisenniveau erreichen (IMF, 2021).

Vielfach werden Mobilitätsdaten verwendet, um die Auswirkungen der Pandemie auf die Wirtschaftstätigkeit zeitnah und mit hoher Frequenz abzuschätzen. Die verfügbaren Daten für Afrika zeigen, dass die Mobilität in allen Ländern in den ersten Monaten der Pandemie erheblich eingeschränkt wurde, als zum ersten Mal ShutdownMaßnahmen verhängt wurden und außerdem viele Menschen freiwillig ihr Verhalten änderten, um das Infektionsrisiko zu verringern. Am drastischsten ging die Mobilität in Nigeria, Ruanda und Südafrika zurück, wo sie zwischen $40 \%$ und $70 \%$ niedriger war als vor der Pandemie (vgl. Abbildung 3). In den meisten Ländern wurden die Auswirkungen im Laufe der Zeit geringer. Im Sommer 2021 ging die Mobilität im Zuge einer dritten Pandemiewelle erneut zurück. Anfang August meldete die Weltgesundheitsorganisation einen neuen Höchststand an wöchentlichen Todesfällen in Zusammenhang mit COVID-19 auf dem Kontinent. Das Auftreten neuer Coronavirus-Varianten und niedrige Impfraten gelten als Hauptgründe für den erneuten Anstieg der Infektionen und Todesfälle.
Abbildung 3

Mobilität in ausgewählten afrikanischen Ländern

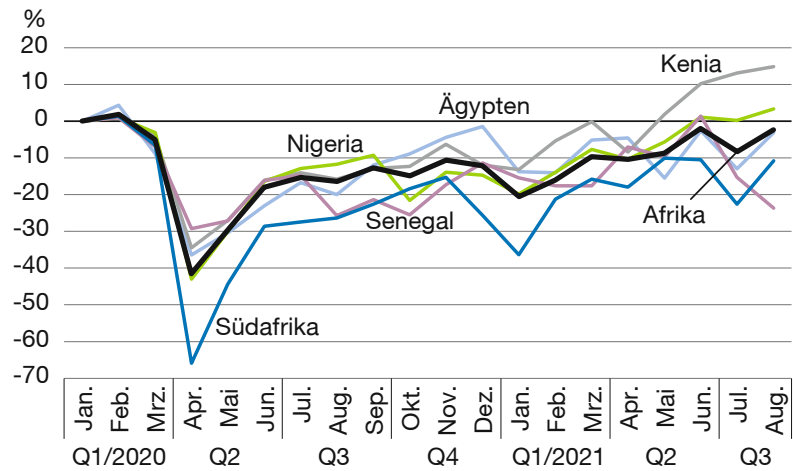

Anmerkungen: Prozentuale Abweichung vom Vorkrisenniveau. Monatsdurchschnitte. Letzter enthaltener Wert: 23.08.2021. Aggregat Afrika: BIP-gewichteter Durchschnitt aller Länder, für die Daten verfügbar sind.

Quelle: Google (2021); Berechnungen des IfW Kiel.

Im Vergleich zu anderen Weltregionen ist die Impfrate mit etwa $5 \%$ immer noch dramatisch niedrig. 42 der 54 afrikanischen Staaten werden wohl das Ziel verfehlen, bis Ende September 2021 die am stärksten gefährdeten $10 \%$ der Bevölkerung eines jeden Landes zu impfen (WHO, 2021). Zwar stabilisierte sich die Situation zuletzt wieder etwas und die Mobilitätsindikatoren deuten auf eine Erholung in den meisten Ländern nach der dritten Welle hin. Zugleich hat sich das weltwirtschaftliche Umfeld seit der zweiten Jahreshälfte 2020 wieder deutlich verbessert. Um die weitere wirtschaftliche Erholung zu sichern und damit längerfristige Folgen für die Entwicklung Afrikas zu vermindern, ist aber ein höheres Impftempo - und damit die Unterstützung der internationalen Gemeinschaft - erforderlich.

Klaus-Jürgen Gern; Saskia Meuchelböck klaus-juergen.gern@ifw-kiel.de; saskia.meuchelboeck@ifw-kiel.de

\section{Literatur}

Bloomberg (2021), One in Four People in Africa's Biggest City May Have Had Covid, 23. Februar.

Gern, K.-J., O. Lück und S. Meuchelböck (i.E.), Covid-19 in Africa and its impact on the economy, Kiel Policy Brief, im Erscheinen.

Google (2021), Covid-19 Mobility Report (Workplace mobility), https:// www.google.com/covid19/mobility/ (8. September 2021).

IMF (International Monetary Fund) (2021), Regional Economic Outlook. Sub-Saharan Africa - Navigating a Long Pandemic, April.

Mahler, D., N. et al. (2021), Updated estimates of the impact of COVID-19 on global poverty: Turning the corner on the pandemic in 2021?, World Bank Blogs, 6. September.

Tessema, S. und J. Nkengasong (2021), Understanding COVID-19 in Africa, Nature Reviews Immunology, 21, 469-470.

The Economist (2021), The pandemic's true death toll, 2. September. WHO (World Health Organization) (2021), Eight in 10 African countries to miss crucial COVID-19 vaccination goal, 2. September.

Weltbank (2021), World Development Indicators. 